\title{
OUTCOME OF MITOCHONDRIAL DISEASES
}

Researchers at Children's Hospital, University Medical Center Ljubljana, Slovenia; Molecular Neurogenetics, Milan, Italy; and Paracelsus Medical University, Salzburg, Austria, evaluated the functional outcome of 22 children with mitochondrial diseases. Twelve children (55\%) had pyruvate dehydrogenase complex deficiency, 4 had Leigh syndrome, and 10 had respiratory chain enzyme deficiencies. Complex IV was the most frequent enzyme deficiency. The morbidity was high, but the mortality was low (18\%). Total Mitochondrial Disease Criteria score was above 7, $82 \%$ above 8 . Using the Pediatric Evaluation of Disability Inventory and scaled scores, a large variability in functional skills was seen in self-care, mobility, and social function. Also, a large variability in caregiver assistance, from independence to total dependence on assistance was observed. Few patients used assistive devices. Children with mitochondrial disease have severe limitations in cognition and communication and are dependent on their parents. Functional status is a necessary part of the evaluation of children with mitochondrial disease. (Rogac M, Meznaric M, Zeviani M, Sperl W, Neubauer D. Functional outcome of children with mitochondrial diseases. Pediatr Neurol April 2011;44:340-346). (Respond: Dr Rogac. E-mail: mihael.rogac@mf.uni-lj.si).

COMMENT. Evaluation of skills in self-care, mobility, and social function is important in the management of children with mitochondrial disease. The Pediatric Evaluation of Disability Inventory is an effective bedside measure of estimating a child's need for caregiver assistance and assistive devices.

\section{INFECTIOUS DISORDERS}

\section{POTT'S PARAPLEGIA IN AN INFANT}

Neurosurgeons at the Barrow Neurological Institute, St Joseph's Hospital, Phoenix, AZ, report a 13-month-old Hispanic boy who presented with paraplegia caused by tuberculosis affecting the mid-thoracic spine. The infant walked at 9 months, and at 11 months, he and his family were diagnosed with pulmonary TB. Despite a full course of anti-TB medications, the boy developed a progressive paraplegia. He was spastic and hyperreflexic, and ankle clonus and Babinski signs were elicited. A kyphotic gibbus deformity was revealed on MRI, and with contrast, a paraspinal and epidural abscess was seen from T2 to T6, ventral, extending into the spinal canal, and compressing the spinal cord with displacement to the right. CT spine showed complete destruction of T4 vertebral body and collapse of T3 onto the body of T5. Surgery involved laminectomy, corpectomies from T2 to T4 via posterior approach, drainage of abscess, Steinmann pins, and grafts from T1 to T6. He was continued on antibiotics for 4 months and at 26 months after surgery, he could walk 5 steps and had no progression of the kyphotic deformity. (Consigilieri G, Kakarla UK, Theodore N. Pott disease in a 13-month-old: Case-report. Neurosurgery May 2011;68(5):E1485-E1490). (Respond: Nicholas Theodore MD, FACS, Barrow Neurological Institute, $350 \mathrm{~W}$ Thomas Rd, Phoenix, AZ 85013. E-mail: neuropub@chw.edu). 